\title{
BMJ Open A randomised controlled trial of an intervention to facilitate the implementation of healthy eating and physical activity policies and practices in childcare services
}

\author{
Jannah Jones, ${ }^{1,2,3,4}$ Luke Wolfenden, ${ }^{1,2,3,4}$ Rebecca Wyse, ${ }^{1,2,3,4}$ \\ Meghan Finch, ${ }^{1,2,3,4}$ Sze Lin Yoong, ${ }^{2,3,4}$ Pennie Dodds, ${ }^{2,3}$ Nicole Pond, ${ }^{1,3}$ \\ Karen Gillham, ${ }^{1,3}$ Megan Freund, ${ }^{1,2,3,4}$ Patrick McElduff, ${ }^{2}$ Paula Wye, ${ }^{1,2,3}$ \\ John Wiggers ${ }^{1,2,3,4}$
}

To cite: Jones J,

Wolfenden L, Wyse R, et al. A randomised controlled trial of an intervention to facilitate the implementation of healthy eating and physical activity policies and practices in childcare services. BMJ Open 2014:4:e005312.

doi:10.1136/bmjopen-2014005312

- Prepublication history for this paper is available online. To view these files please visit the journal online (http://dx.doi.org/10.1136/ bmjopen-2014-005312).

Received 21 March 2014 Accepted 27 March 2014

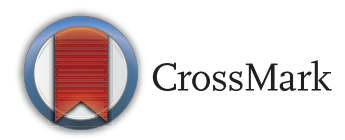

For numbered affiliations see end of article.

Correspondence to Jannah Jones; jannah.jones@ hnehealth.nsw.gov.au

\section{ABSTRACT}

Introduction: Childhood overweight and obesity tracks into adulthood, increasing the risk of developing future chronic disease. Implementing initiatives promoting healthy eating and physical activity in childcare settings has been identified as a priority to prevent excessive child weight gain. Despite this, few trials have been conducted to assess the effectiveness of interventions to support population-wide implementation of such initiatives. The aim of this study is to assess the effectiveness of a multicomponent intervention in increasing the implementation of healthy eating and physical activity policies and practices by centre-based childcare services.

Methods and analysis: The study will employ a parallel group randomised controlled trial design. A sample of 128 childcare services in the Hunter region of New South Wales, Australia, will be recruited to participate in the trial. 64 services will be randomly allocated to a 12-month implementation intervention. The remaining 64 services will be allocated to a usual care control group. The intervention will consist of a number of strategies to facilitate childcare service implementation of healthy eating and physical activity policies and practices. Intervention strategies will include implementation support staff, securing executive support, consensus processes, staff training, academic detailing visits, performance monitoring and feedback, tools and resources, and a communications strategy. The primary outcome of the trial will be the prevalence of services implementing all healthy eating and physical activity policies and practices targeted by the intervention. To assess the effectiveness of the intervention, telephone surveys with nominated supervisors and room leaders of childcare services will be conducted at baseline and immediately postintervention.

Ethics and dissemination: The study was approved by the Hunter New England Human Research Ethics Committee and the University of Newcastle Human

\section{Strengths and limitations of this study}

- The study has a strong design incorporating random allocation and blinding of data collection interviewers.

- The intervention consists of a broad set of intervention strategies and is based on a theoretical framework and past research evidence.

- The study is one of only a handful of randomised trials of such interventions internationally, and the first of its kind in Australia.

Research Ethics Committee. Study findings will be disseminated widely through peer-reviewed publications and conference presentations.

Trial registration number: Australian Clinical Trials Registry ACTRN12612000927820.

\section{INTRODUCTION}

Globally the prevalence of child overweight and obesity is increasing, with $9 \%$ of children aged $0-5$ years expected to be overweight or obese by the year 2020. ${ }^{1}$ Overweight and obesity in childhood tracks into adulthood, ${ }^{2}$ increasing the risk of developing a number of chronic health conditions. Given early childhood represents a critical period in the development of diet and physical activity behaviours, interventions that aim to improve such behaviours have been recommended to prevent the onset of obesity among young children. ${ }^{3-7}$

Childcare services have been identified as a key setting to create environments supportive of child healthy eating and physical activity. 
In developed countries, childcare services provide access to a large and growing number of children for prolonged periods each day. ${ }^{8-11}$ Evidence from randomised and quasi-experimental trials suggests that healthy eating and physical activity promoting policies and practices can increase time children spend being physically active, improve child fundamental movement skill proficiency, improve child diet quality in care and prevent excessive weight gain. ${ }^{12-15}$ As such, licensing and accreditation requirements and best practice guidelines for the childcare setting recommend that services implement a number of policies and practices to support healthy eating and physical activity. ${ }^{16-21}$

Despite such evidence and recommendations from best practice guidelines, childcare service implementation of healthy eating and physical activity policies and practices appears to be limited internationally ${ }^{17}$ and within Australia. ${ }^{22}$ Australian research, for example, suggests that just $48-50 \%$ of centre-based childcare services have a written physical activity policy, $46-60 \%$ programme time each day for fundamental movement skill development, while 25-28\% provide opportunities each day for children to participate in sedentary screen time, ${ }^{22}$ and $60 \%$ of Australian preschooler lunchboxes contain more than one serve of high fat, salt or sugar foods or drinks. ${ }^{23}$

Without adequate implementation, the potential public health benefits of interventions to prevent childhood overweight and obesity in childcare services will not be realised. An agency for healthcare research and quality systematic review, however, identified no trials examining the effectiveness of interventions to improve childcare service implementation of healthy eating and physical activity promoting policies and practices. ${ }^{24}$
Further, trials published since the review search period have reported non-significant improvements or improvements in only a minority of healthy eating or physical activity policies of practices targeted by the intervention. ${ }^{13} 14{ }^{25-27}$ Previous trials have also utilised a narrow set of implementation strategies (eg, workforce development and resource provision) $)^{14} 28$ or developed interventions without the guidance of conceptual or theoretical frameworks, ${ }^{13} 28$ limiting their potential effectiveness. Finally, most previous trials have been conducted on small numbers of childcare services (less than 20) limiting their capacity to guide approaches aimed at achieving population-level service improvements. ${ }^{27} 28$

Given the limitations of the existing evidence base, a randomised trial of a theoretically based, multicomponent implementation intervention to increase the implementation of healthy eating and physical activity policies and practices in childcare services in Australia will be conducted. This paper describes the protocol by which this trial will be conducted.

\section{METHODS AND ANALYSIS \\ Study aim}

The aim of this study is to assess the effectiveness of multicomponent intervention in increasing the implementation of healthy eating and physical activity policies and practices by centre-based childcare services.

\section{Study design}

The study will employ a parallel group randomised controlled trial design (see figure 1). A sample of eligible childcare services in the study region will be randomly selected and invited to participate in the trial. Sixty-four
Figure 1 CONSORT flow diagram estimating the progress of childcare services through the trial.

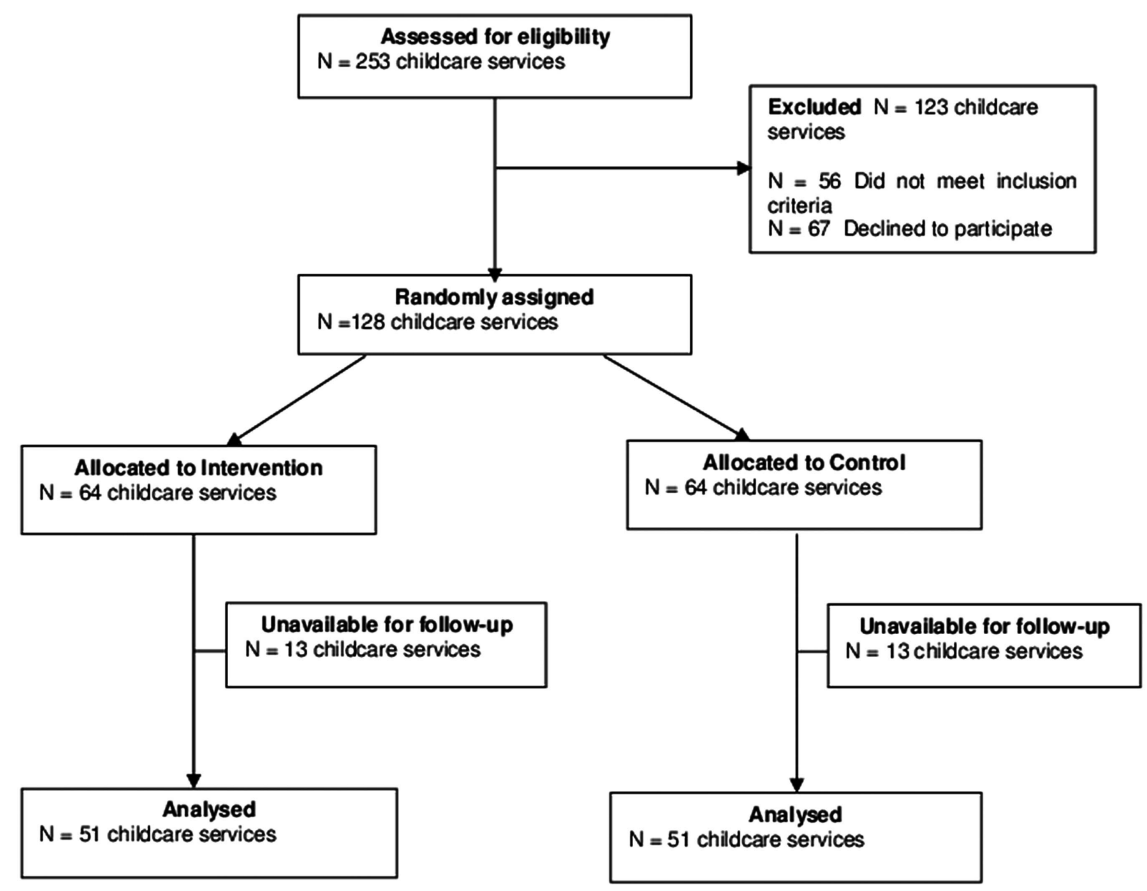


services will be allocated to receive a 12-month implementation intervention, and 64 services will be allocated to receive usual care and will serve as a control group. To assess the effectiveness of the intervention, across each service, surveys administered via computer assisted telephone interview (CATI) will be conducted with the nominated supervisor (service manager) and room leader (room supervisor) caring for children 3-5 years. The surveys will be conducted at baseline and immediately postintervention. CATI interviewers will be blind to group allocation.

The research will be reported in accordance with the requirements of CONSORT Statement. ${ }^{29}$ The trial is funded by the Australian National Preventive Health Agency (reference: 95WOL2011) with in-kind support for the trial provided by Hunter New England Population Health.

\section{Research setting}

The study will take place in the Hunter region of New South Wales, Australia. The region encompasses nonmetropolitan 'major cities' and 'inner regional' areas as described by the Australian Statistical Geography Standard. ${ }^{30}$ There are 586000 people residing in the area, of which 23000 are children aged 3-5 years. ${ }^{31} 32$ Approximately $3 \%$ of residents are of Aboriginal or Torres Strait Islander origin and 4\% speak languages other than English. ${ }^{31}{ }^{32}$ The Hunter region has lower indices of socioeconomic status than the New South Wales state average. ${ }^{31} 32$

\section{Sample}

\section{Childcare services}

For the purpose of the study, centre-based childcare services include preschools and long day care services. More than $60 \%$ of Australian children aged 3-5 years attend preschool or long day care. ${ }^{11}$ In New South Wales, preschools provide centre-based care for $6-8 \mathrm{~h}$ / day and enrol children aged between 3-6 years. Long day care services provide centre-based care for eight or more hours per day and usually enrol children aged from 6 weeks up to 6 years. Both types of services provide specific programmes for children aged 3-5 years that include educational and developmental activities to assist children in their preparation for school. ${ }^{33}$ Across Australian states and territories, the role and function of preschools and long day care services are similar and licensing and accreditation requirements regarding healthy eating and physical activity policies and practices are identical. ${ }^{21}$ Physical activity policies and practices are similar across both service types. ${ }^{22}$

In 2012, there were over 250 centre-based childcare services in the study region. A current list of all childcare services in the region provided by the New South Wales Ministry of Health will serve as the sampling frame for the study. Services catering exclusively for children requiring specialist care will be excluded from the trial (representing less than $1 \%$ of services in the region), as will services that provide on-site meals to children (representing approximately $30 \%$ of services in the region). Government funded, Department of Education services (representing approximately $3 \%$ of services in the region) will also be excluded as ethics approval has not been obtained from the relevant government department.

\section{Nominated supervisors and room leaders}

Within participating childcare services, nominated supervisors and room leaders will be invited to participate in CATI surveys to assess the effectiveness of the intervention. The nominated supervisor is the person responsible for the management of the service and will be asked to report on the implementation of whole-of-service policies and practices. The room leader caring for 3-5-year-old children is the head teacher of the classroom and will be asked to report on the implementation of specific healthy eating and physical activity policies and practices within their room. Where there are more than one nominated supervisors or room leaders within a service, a member of the research team will randomly select one staff member to be invited to participate by requesting the staff member with the most recent birthday. There are no exclusion criteria for nominated supervisor or room leader participation.

\section{Recruitment procedures}

In order to maximise service participation in the study, a recruitment strategy will be employed based on a review of effective recruitment practices within the school setting ${ }^{34}$ which has also been successfully executed in childcare settings by the research team. ${ }^{35-37}$ Specifically, one member of the research team will act as a dedicated recruitment coordinator. The coordinator will manage the recruitment of services into the trial and monitor consent rates. Services will be provided with the direct phone number of the coordinator for enquiries regarding the research. Study information will be mailed to childcare services inviting participation in the study. Nominated supervisors will be invited to provide consent for their service participation in the study, consent for their participation in a CATI survey and consent to contact a room leader to participate in a CATI survey. Two weeks after receipt of the information, nominated supervisors and room leaders will be telephoned by a research assistant who will confirm eligibility, invite study participation and schedule a time to conduct the baseline CATI surveys. To minimise attrition, prior to follow-up data collection, services will receive letters thanking them for their participation to date and reminding them that they will be telephoned again to participate in follow-up data collection. ${ }^{38}$

\section{Random allocation of childcare services}

Following the completion of baseline data collection, childcare services will be randomly allocated to either the intervention or control condition using a 
computerised random number function in Microsoft Excel. Allocation of services will be undertaken by a statistician who will have no other involvement in the trial. The random allocation of childcare services will not be stratified, given previous studies have found only slight differences in implementation of healthy eating and physical activity policies and practices by service type (preschool or long day care service), size or the socioeconomic or geographic characteristics of the service locality. ${ }^{22}$ Services will be randomised in a 1:1 (intervention:control) ratio. Owing to the difficulty in blinding services to their group allocation, this trial will be an 'open' trial and services will not be blind to study allocation.

\section{Intervention}

Implementation intervention

The intervention was designed by an expert advisory group of health promotion practitioners, psychologists, dietitians, behavioural scientists and physical activity experts, in consultation with nominated supervisors from local childcare services.

The intervention will seek to increase service implementation of healthy eating and physical activity policies and practices. The policies and practices are consistent with best practice Australian healthy eating and physical activity guidelines for the childcare setting, ${ }^{39} 40$ and evidence reviews of policies and practices shown to be associated with child healthy eating and physical activity. ${ }^{17} 19$ Specifically the intervention will target the implementation of the policies and practices described in the measures section.

\section{Intervention strategies}

The intervention will consist of a number of strategies to facilitate childcare service implementation of healthy eating and physical activity policies and practices. The strategies to support implementation have been developed to address identified barriers reported by childcare service staff to implementing health promoting policies and practices. ${ }^{41}$ The effectiveness of the intervention components are supported by reviews of implementation and practice change interventions, ${ }^{42-45}$ and are consistent with recommendations for scaling up health programmes. ${ }^{46}$

Specifically, the intervention will include the following strategies:

1. Implementation support staff ${ }^{47}$ : Each service will be allocated an individual implementation support staff member who will provide ongoing implementation support and positive reinforcement to the service during the 12-month intervention. Support will be provided via face-to-face visits, telephone and email contacts. ${ }^{47}$ Each contact will draw on continuous quality improvement principles to review progress, provide positive reinforcement and facilitate reflection, problem solving, goal setting and action planning. ${ }^{47}{ }^{48}$ Services will be allocated to staff members at a ratio of 20 services for every one full-time equivalent staff member.

2. Securing executive support ${ }^{49}$ : To secure executive support and leadership, the importance and benefits of implementing healthy eating and physical activity policies and practices will be communicated by implementation support staff to nominated supervisors during telephone contact, face-to-face meetings, training workshops, service visits and newsletters. ${ }^{49}$ Nominated supervisors will be supported to monitor the implementation of policies and practices and will be asked to demonstrate leadership and managerial support, by leading the development and implementation of nutrition and physical activity policies, communicating expectations to childcare service staff during staff meetings, cofacilitating training workshops with implementation support staff and participating in consensus processes during staff training workshops. Such factors have been associated with implementation success. ${ }^{48-50}$

3. Employment of consensus processes 55152 : Consensus processes will be employed to reduce childcare service staff resistance to policy and practice change by resolving any discrepancies between existing standards and expectations of childcare service staff. ${ }^{51}$ A consensus process will occur following each staff training workshop where implementation support staff will facilitate a discussion involving nominated supervisors and childcare service staff, to reach agreement and a group decision regarding an implementation strategy for the targeted policies and practices. ${ }^{51}$ The discussion will involve all childcare service staff and will allow for sharing of information, group goal setting, problem solving of barriers that may impede implementation and action planning. ${ }^{45} 5152$

4. Provision of staff training ${ }^{53}$ : A series of three $1 \mathrm{~h}$ training workshops will be provided to childcare service staff at their service, typically outside of operating hours. The workshops will introduce key healthy eating and physical activity intervention messages and will focus on introducing and supporting the implementation of the healthy eating and physical activity policies and practices. The workshops will combine didactic and interactive components, including opportunities to practice new skills and to role-play, have group discussions, discuss case studies and receive facilitator provided feedback, as such elements have been suggested to be more effective than didactic education alone. ${ }^{45}$ 53-55 Training workshops will be facilitated by experienced implementation support staff with expertise in early childhood education and health promotion and will be tailored to address the specific policies or practices that childcare services are not currently implementing.

5. Provision of academic detailing visits ${ }^{48}$ : On three occasions following each training workshop, implementation support staff will conduct academic detailing visits with childcare service staff at their service. The 
visits will draw on the principles of academic detailing outlined by Soumerai and Avon ${ }^{48}$ and will involve support staff observing childcare service staff implementation of practices targeted by the intervention. During the visits, implementation support staff will provide immediate feedback based on observations of practice regarding policy or practice implementation, provide further advice or support as required and reinforce observed practice improvements. Subsequent visits will be similarly structured by tailoring based on the policies and practices that services are having difficulty implementing. ${ }^{56}$

6. Provision of tools and resources ${ }^{47}$ : All services will receive a package of tools and resources to support and sufficiently equip childcare service staff to implement the healthy eating and physical activity policies and practices. The resources were reviewed by local nominated supervisors and childcare service staff to ensure that they are relevant, attractive and user friendly in order to increase the likelihood of use. ${ }^{47}$ Specifically the resource package includes an implementation manual, policy templates, reflection and goal-setting document, self-assessment forms, a DVD with age appropriate physical activity games to encourage the development of fundamental movement skills, lanyards to be worn by childcare service staff during outdoor play with pictures and instructions for each fundamental movement skill, healthy food guideline posters and parent lunchbox resources. Resources will be provided to services in electronic and hard copy forms.

7. Provision of performance monitoring and feedback ${ }^{57}{ }^{58}$ : Performance feedback will be delivered by implementation support staff to nominated supervisors and childcare service staff members. Data for the performance feedback will be compiled initially using information collected via the baseline CATI and thereafter project records maintained by implementation support staff. Feedback will describe individual service progress towards implementation of the targeted healthy eating and physical activity policies and practices at six intervals throughout the 12-month intervention. Feedback will be provided in written, graphic and verbal modalities. ${ }^{57}{ }^{58}$ In order to maximise the effectiveness of this strategy, performance will be compared against agreed benchmarks and the performance of all services receiving the intervention. Action plans will be negotiated with services to facilitate further performance improvement. ${ }^{57}$

8. Employment of a communications strategy ${ }^{59}$ : Services will receive bimonthly printed newsletters which will communicate key messages relating to the healthy eating and physical activity policies and practices. ${ }^{59}$ The newsletters will case study individual services which have undertaken successful and innovative approaches to implementation, and include content to help overcome key implementation barriers. Services that demonstrate achievement of all healthy eating and physical activity policies and practices will also receive a certificate of recognition and will be promoted to other services through newsletters and staff training workshops. ${ }^{47}$ Nominated supervisors will also be able to nominate individual childcare service staff members who demonstrate significant achievement towards the key policies and practices for a certificate of recognition.

\section{Theoretical perspective}

The use of comprehensive theoretical frameworks, particularly those operating at multiple levels within complex systems, has been recommended to guide the development of implementation interventions. ${ }^{24}$ Given the lack of evidence for the effectiveness of theoretical frameworks for community-based implementation interventions, ${ }^{24}$ the intervention draws on implementation theory previously utilised in clinical settings. ${ }^{24}$ Specifically, the intervention draws on Damschroder's consolidated framework for implementation research. ${ }^{60}$ The framework integrates 19 theoretical models and is composed of five major domains identified as influential in successful intervention implementation: intervention characteristics, outer setting, inner setting, characteristics of the individuals involved and the process of implementation. The constructs contained within the five domains of the consolidated framework for implementation research and the application of the relevant constructs to the intervention are described in table 1 .

Intervention personnel, training, supervision and monitoring Implementation support staff will attend a 2-day training workshop which will focus on equipping staff with sufficient knowledge and skills to deliver the intervention. Intervention delivery will be overseen by a project manager. All implementation support staff will participate in fortnightly group meetings, where a selfregulatory model of peer supervision will be utilised to facilitate learning, improve staff performance and help standardise intervention delivery. ${ }^{61}$ Project records will be maintained by implementation support staff and will be used to monitor intervention delivery according to the protocol. Such staff will also have access to dietitians, psychologists and childcare service staff for specialist advice and support.

\section{Control group}

Participating services randomised to the control group will not receive any intervention by the research team during the study period.

\section{Data collection procedures}

Baseline and follow-up data will be collected through two CATI surveys-one administered to the nominated supervisor and one administered to the room leader caring for 3-5-year-old children at all participating services. Similar surveys have been used previously by the 
Table 1 Application of the consolidated framework for implementation research

\section{Construct \\ Application to intervention}

Intervention characteristics: characteristics of the healthy eating and physical activity policies and practices

Intervention source: Was the intervention developed internally The intervention was designed externally by an expert by childcare services or by external agencies?

advisory group of health promotion practitioners,

psychologists, dietitians, behavioural scientists and physical activity experts, in consultation with nominated supervisors from local childcare services. Components of the intervention (including staff training workshops and the tools and resources) will be pilot tested with childcare services prior to implementation

There is strong evidence to support childcare services as a setting to create environments more supportive of child healthy eating and physical activity. The strength of evidence will be communicated to childcare service staff during telephone contact, face-to-face meetings, training workshops, service visits and newsletters. Policies and practices are consistent with licensing and accreditation requirements and evidence-based best practice guidelines for the childcare setting

Adaptability: Is the intervention able to be adapted or tailored to meet the needs of the childcare service?

Complexity: What are the nominated supervisor's and childcare service staff's perceptions of implementation difficulty?

Design quality and packaging: What are the nominated supervisor's and childcare service staff's perceptions of how the intervention is presented?

Cost: What are the costs of the intervention and associated implementation?

Outer setting: the economic, political and social context within which the childcare service operates

Peer pressure: Is there competitive pressure to implement the intervention?

External policy and incentives: What are the external strategies to spread the intervention (including policy and regulations, external mandates, recommendations and guidelines)?
Staff training workshops will be tailored to meet service needs based on the policies and practices not being implemented at baseline. Implementation support staff will employ consensus processes regarding how to implement policies and practices that best suit the service needs. Advice on the use of tools and resources will be tailored based on identified barriers to changing healthy eating and physical activity policy and practice

Healthy eating and physical activity policies and practices endorsed by the intervention are consistent with existing licensing and accreditation requirements for the setting. Support will focus on integrating policies and practices into existing service routines to reduce the burden on childcare service staff. This will be communicated to childcare service staff during telephone contact, face-to-face meetings, training workshops, service visits and newsletters

Tools and resources will be reviewed by nominated supervisors and childcare service staff to ensure that they are professionally presented, attractive and user friendly. Newsletters will be bright and visually appealing The intervention will be provided to childcare services at no cost. This will be communicated to childcare service staff during telephone contact, face-to-face meetings, training workshops, service visits and newsletters

Successful implementation of all policies and practices by childcare services will be communicated to other intervention services using a communications strategy. Individual service performance will be compared against the performance of all services receiving the intervention to permit peer comparison Policies and practices are consistent with licensing and accreditation requirements and evidence-based best practice guidelines for the childcare setting. The use of continuous quality improvement principles during support contacts aligns with external policy and accreditation requirements for the childcare setting 
Table 1 Continued

Construct Application to intervention

Inner setting: the childcare service structural, political and cultural contexts through which the implementation process will proceed

Tension for change: Do the nominated supervisor and childcare service staff perceive the current situation as needing to change?

Relative priority: Do childcare service staff have a shared perception of the importance of implementation within the childcare service?

Organisational incentives and rewards: Does the intervention include incentives such as goal-sharing awards, performance reviews, increased stature?

Goals and feedback: Are goals clearly communicated, acted on and fed back to nominated supervisor and childcare service staff?

Leadership engagement: Are nominated supervisors committed, involved and accountable for the implementation?

Access to information and knowledge: How easy is it for childcare service staff to access information and knowledge about the intervention and how to incorporate it into work tasks?

Characteristics of individuals: characteristics of childcare service staff

Knowledge and beliefs about the intervention: What are the nominated supervisor and childcare service staff attitudes towards the intervention?

facilitate implementation

Process: the active change process aimed to achieve individual staff and childcare service use of the intervention Engaging: Are appropriate individuals involved in the implementation through education, role modelling, training?
The need for change will be explored during consensus processes, communicated to childcare service staff through newsletters via presentation of data and advocated by implementation support staff at training workshops and other service contacts

Nominated supervisors will communicate to childcare service staff that implementation of the targeted policies and practices represent an organisational priority during training workshops and staff meetings and via the development and endorsement of supportive policy

Services that demonstrate achievement of all policies and practices will receive a certificate of recognition and will be promoted to other intervention services in newsletters and through training workshops

Implementation support staff contacts with childcare service staff will draw on continuous quality improvement principles to review progress, provide positive reinforcement, discuss deficits identified from self-monitoring and facilitate reflection, problem solving, goal setting and action. Furthermore, performance feedback will be provided to services regarding achievement of key implementation goals

Nominated supervisors and management committees will be engaged and encouraged to communicate their endorsement of practice changes to childcare service staff during staff meetings, training workshops and via the consensus processes. Performance feedback reports will be circulated to management committees and childcare service staff Nominated supervisors and childcare service staff will receive resources and ongoing support from implementation support staff via face-to-face meetings, telephone and email.

Services will also be able to contact implementation support staff at any time for advice or assistance

Beliefs of nominated supervisors and childcare service staff will be explored during initial service contacts. Beliefs which are incongruent with policy or practice implementation will be targeted during future intervention contacts. A focus of training workshops will be to provide knowledge for childcare service staff to motivate and equip staff with knowledge to Service management committees, nominated supervisors and childcare service staff will be engaged in implementation through involvement in discussions regarding service priorities, service goals and strategies to meet goals and overcome barriers. Services will be encouraged to explain and communicate any changes to service operation to families research team to assess the policies and practices of childcare services. $^{22} 2535$ The CATI survey will take approximately 15-25 min to complete. CATI interviewers will be provided with training to ensure they understand and adhere to data collection protocols. Interviewers will also be required to practice the survey script prior to administration. Interviewers will not participate in the delivery of the intervention and will be blind to service group allocation. Baseline data will be collected 1-2 months prior to start of intervention delivery. The survey administered at baseline will be repeated immediately postintervention. 


\section{Measures}

Childcare service operational characteristics

Operational information will include the number of years in operation, the number of enrolled and attending children aged 3-5 years, the type of service (preschool or long day care service), number of primary contact teaching staff and hours of operation. Items to assess operational characteristics will be similar to those used in other Australian surveys of childcare services. ${ }^{22} 2535$

\section{Service healthy eating and physical activity policies and practices}

The primary outcome is the change in prevalence of services meeting all healthy eating and physical activity policies and practices. Given there are no validated items to assess such policies and practices in Australian childcare services, survey items assessing healthy eating and physical activity policies and practices will be based on (where available) those previously used in the description of the obesity prevention environments of Australian childcare services, ${ }^{22} 2535$ and validated items from the USA Nutrition and Physical Activity Self-Assessment for Child Care instrument ${ }^{6263}$ and the Child Care Nutrition and Physical Activity Assessment Survey. ${ }^{64}$ The validated items have adequate agreement (A) and $\kappa$ scores $(\mathrm{K})$. The items will assess:

1. Presence of written nutrition $(\mathrm{K}=0.76 ; \mathrm{A}=88.1 \%)$ and physical activity policies $(\mathrm{K}=0.79 ; \mathrm{A}=91 \%)$;

2. Staff monitoring of children's lunchboxes against written nutritional guidelines $(\mathrm{A}=88 \%)$ and provision of feedback to parents when a non-compliant food is packed (statistic unavailable);

3. Provision of water $(\mathrm{A}=82 \%)$ or reduced fat milk only to children $(\mathrm{K}=0.73 ; \mathrm{A}=82.1 \%)$;

4. Staff role modelling of physically active play $(\mathrm{K}=0.59$; $\mathrm{A}=69.1 \%)$ and healthy eating $(\mathrm{A}=90 \%)$;

5. Staff provision of prompts and positive comments to children to encourage physical activity (statistic unavailable) and healthy eating (statistic unavailable);

6. Provision of adult guided fundamental movement skill development activities (statistic unavailable);

7. Restriction of sedentary screen time $(\mathrm{A}=97 \%)$.

\section{Intervention acceptability}

At follow-up, the CATI survey for intervention services will include items assessing the use, acceptability and satisfaction with the intervention resources, training and support provided to services. The items will require nominated supervisors and room leaders to respond to a series of statements on a four-point Likert scale ranging from 'strongly agree' to 'strongly disagree'. Acceptability items will be developed by the research team based on previous assessments of staff acceptability in delivering health promotion programmes. ${ }^{25}$
Context

For descriptive purposes and to aid an assessment of the external validity of the trial findings, a systematic search will be conducted to describe the context in which the trial was conducted. Specifically the search will include local news archives, websites of national and New South Wales health and education departments, accreditation standards and professional healthy eating and physical activity guidelines to identify the existence of or changes in government policy, standards, funded programmes or guidelines that may influence the healthy eating and physical activity environments of childcare services. The search will include the 12 months prior to and 12 months during the intervention delivery.

\section{Sample size and power calculations}

A $20 \%$ study attrition rate is estimated based on the previous research in this setting. ${ }^{25}$ Given this, recruitment of 128 services into the trial at baseline would be sufficient to provide follow-up data from approximately 102 childcare services (51 per group) and enable the detection of an absolute difference between groups in the prevalence of services meeting all policies and practices of $27 \%$ with $80 \%$ power and an $\alpha$ value of 0.05 (as outlined in figure 1). The sample size calculation is based on an expected prevalence of control group services meeting all policies and practices at follow-up of $25 \%$ (unpublished data).

\section{Statistical analysis}

Descriptive statistics will be calculated to describe the demographic and service characteristics of intervention and control groups at baseline. All statistical tests will be 2-tailed with an $\alpha$ value of 0.05 . The primary trial outcome will be analysed under an intention-to-treat framework where services are analysed based on the groups to which they were allocated, regardless of the treatment type or exposure received. ${ }^{65}$ Intervention effectiveness will be determined using a logistic regression model adjusted for baseline values. Consistent with the approach of White $e t a l,{ }^{66}$ the primary analysis will use all available data and include baseline policy and practice implementation as a covariate. A sensitivity analysis will be performed by imputing baseline observations at follow-up for missing data. Subgroup analyses will also be conducted by comparing service type (preschool or long day care service), socioeconomic characteristics (service postcode in the top or bottom $50 \%$ of New South Wales according to the Socioeconomic Indices for Areas) and geographic characteristics (service locality, classified as urban or rural areas according to the Australian Statistical Geography Standard).

\section{DISCUSSION}

There is a clear need for intervention studies to extend the research regarding the implementation of 
healthy eating and physical activity policies and practices by childcare services. This trial aims to advance the currently limited evidence in this field and will contribute important information regarding the effectiveness of intervention strategies to facilitate the population-wide implementation of healthy eating and physical activity policies and practices in childcare services.

Strengths of this study include the trial's randomised design, the use of theoretical framework and broad set of intervention strategies, the long duration of the intervention (12 months), the implementation of procedures to reduce potential threats to internal validity, such as the blinding of data collection interviewers and computer-based randomisation of groups.

\section{CONCLUSION}

This manuscript provides a description of the study methods to be employed as part of a randomised controlled trial of a multicomponent intervention to facilitate the implementation of healthy eating and physical activity policies and practices in childcare services. The study is one of only a handful of randomised trials of such interventions internationally, and the first of its kind in Australia, and will contribute greatly to the evidence regarding the effectiveness of interventions in this setting.

\section{Author affiliations}

${ }^{1}$ Hunter New England Population Health, Wallsend, New South Wales, Australia

${ }^{2}$ School of Medicine and Public Health, University of Newcastle, Callaghan, New South Wales, Australia

${ }^{3}$ Hunter Medical Research Institute, Newcastle, New South Wales, Australia

${ }^{4}$ Priority Research Centre for Health Behaviour, The University of Newcastle,

Callaghan, New South Wales, Australia

Acknowledgements The authors wish to thank members of the Good for Kids. Good for Life Early Childhood Education and Care advisory group.

Contributors $\mathrm{JJ}$ led the development of the manuscript. LW conceived the intervention concept. All the authors contributed to the research design and trial methodology and read and approved the final version of the manuscript.

Funding This study is funded by the Australian National Preventive Health Agency (reference: 95WOL2011) with in-kind support for the trial provided by Hunter New England Population Health. The project also received infrastructure support from the Hunter Medical Research Institute (HMRI).

Competing interests LW secured grant funding from the Australian National Preventive Health Agency.

Ethics approval Ethical approval to conduct the study has been obtained from the Hunter New England Human Research Ethics Committee (reference: 12/08/15/5.01) and the University of Newcastle Human Research Ethics Committee (reference: H-2012-0321).

Provenance and peer review Not commissioned; externally peer reviewed.

Open Access This is an Open Access article distributed in accordance with the Creative Commons Attribution Non Commercial (CC BY-NC 3.0) license, which permits others to distribute, remix, adapt, build upon this work noncommercially, and license their derivative works on different terms, provided the original work is properly cited and the use is non-commercial. See: http:// creativecommons.org/licenses/by-nc/3.0/

\section{REFERENCES}

1. de Onis M, Blossner M, Borghi E. Global prevalence and trends of overweight and obesity among preschool children. Am J Clin Nutr 2010;92:1257-64.

2. Venn AJ, Thomson RJ, Schmidt MD, et al. Overweight and obesity from childhood to adulthood: a follow-up of participants in the 1985 Australian Schools Health and Fitness Survey. Med J Aust 2007; $186: 458-60$.

3. Hesketh KD, Campbell KJ. Interventions to prevent obesity in 05-year-olds: an updated systematic review of the literature. Obesity 2010;18(Suppl 1):P27-35.

4. Goldfield GS, Harvey A, Grattan K, et al. Physical activity promotion in the preschool years: a critical period to intervene. Int $J$ Environ Res Public Health 2012;9:1326-42.

5. Birch LL, Ventura AK. Preventing childhood obesity: what works? Int J Obes (Lond) 2009;33(Suppl 1):P74-81.

6. Swinburn B, Gill T, Kumanyika S. Obesity prevention: a proposed framework for translating evidence into action. Obes Rev 2005;6:23-33.

7. Dietz WH. Critical periods in childhood for the development of obesity. Am J Clin Nutr 1994;59:955-9.

8. Story M, Kaphingst KM, French S. The role of child care settings in obesity prevention. Future Child 2006;16:43-168.

9. Mamedova S, Redford J. Early Childhood Program Participation, from the National Household Education Surveys Program of 2012. Washington, DC: U.S. Department of Education, 2013.

10. Huskinson T, Pye J, Medien K, et al. Childcare and early years survey of parents 2011. UK Department for Education, 2013.

11. Australian Bureau of Statistics. Experimental estimates of preschool education (No. 4240.0). Canberra: Australian Bureau of Statistics, 2011.

12. Trost SG, Fees B, Dzewaltowski D. Feasibility and efficacy of a "Move and Learn" physical activity curriculum in preschool children. $J$ Phys Act Health 2008;5:88-103.

13. Hardy L, King L, Kelly B, et al. Munch and move: evaluation of a preschool healthy eating and movement skill program. Int J Behav Nutr Phys Act 2010;7:80.

14. De Silva-Sanigorski AM, Bell AC, Kremer P, et al. Reducing obesity in early childhood: results from Romp \& Chomp, an Australian community-wide intervention program. Am J Clin Nutr 2010;91:831-40.

15. Adams J, Zask A, Dietrich U. Tooty Fruity Vegie in Preschools: an obesity prevention intervention in preschools targeting children's movement skills and eating behaviours. Health Promot J Austr 2009;20:112-19.

16. Institute of Medicine. Accelerating progress in obesity prevention: solving the weight of the nation. Washington, DC: The National Academies Press, 2012.

17. Larson N, Ward DS, Neelon SB, et al. What role can child-care settings play in obesity prevention? A review of the evidence and call for research efforts. J Am Diet Assoc 2011;111:1343-62.

18. McWilliams C, Ball SC, Benjamin SE, et al. Best-practice guidelines for physical activity at child care. Pediatr 2009;124:1650-9.

19. McPherson ME, Homer CJ. Policies to support obesity prevention for children: a focus on of early childhood policies. Pediatr Clin North Am 2011;58:1521-41.

20. Commonwealth of Australia. Get Up and Grow Healthy Eating and Physical Activity for Early Childhood. 2013. http://www.health.gov.au/ internet/main/publishing.nsf/Content/phd-gug-staffcarers (accessed Mar 2014).

21. Australian Children's Education and Care Quality Authority. National Quality Framework. 2012. http://acecqa.gov.au/national-qualityframework (accessed Mar 2014).

22. Wolfenden L, Neve M, Farrel L, et al. Physical activity policies and practices of childcare centres in Australia. $J$ Paediatr Child Health 2010;47:73-6.

23. Kelly B, Hardy LL, Howlett $S$, et al. Opening up Australian preschoolers' lunchboxes. Aus N Z J Public Health 2010;34:288-92.

24. Rabin BA, Glasgow RE, Kerner JF, et al. Dissemination and implementation research on community-based cancer prevention: a systematic review. Am J Prev Med 2010;38:443-56.

25. Finch $\mathrm{M}$, Wolfenden $\mathrm{L}$, Falkiner $\mathrm{M}$, et al. Impact of a population based intervention to increase the adoption of multiple physical activity practices in centre based childcare services: a quasi experimental, effectiveness study. Int J Behav Nutr Phys Act 2012;9:101.

26. Ward D, Benjamin SE, Ammerman AS, et al. Nutrition and physical activity in child care: Results from an environmental intervention. $A m$ J Prev Med 2008;35:352-6.

27. Pettman T, McAllister M, Verity F, et al. Eat well be active community programs: final report. 2010. http://www.health.sa.gov.au/pehs (accessed Mar 2014). 
28. Williams C, Bollella MC, Strobino BA, et al. "Healthy Start": Outcome of an intervention to promote a heart healthy diet in preschool children. J Am Coll Nutr 2002;21:62-71.

29. Moher D, Schulz KF, Altman DG. CONSORT 2010 statement updated guidelines for reporting parallel group randomised trials. PLoS Med 2010;7:3.

30. Australian Bureau of Statistics. Australian statistical geography standard. Canberra: Australian Bureau of Statistics, 2011. http:// www.abs.gov.au/websitedbs/d3310114.nst/home/australian +statistical+geography+standard+(asgs) (accessed Mar 2014).

31. Australian Bureau of Statistics. 2011 census community profiles Newcastle and Lake Macquarie. Canberra: Australian Bureau of Statistics, 2011. http://www.censusdata.abs.gov.au/census_services/ getproduct/census/2011/communityprofile/111?

opendocument\&navpos=100 (accessed Mar 2014).

32. Australian Bureau of Statistics. 2011 census community profiles Hunter Valley exc Newcastle. Canberra: Australian Bureau of Statistics, 2011. http://www.censusdata.abs.gov.au/census_services/ getproduct/census/2011/communityprofile/106? opendocument\&navpos=100 (accessed Mar 2014).

33. Australian Bureau of Statistics. Childhood education and care June 2008 (Reissue) (No.4402.0). Canberra: Australian Bureau of Statistics, 2009.

34. Wolfenden L, Kypri K, Freund M, et al. Obtaining active parental consent for school-based research: a guide for researchers. Aust $N$ Z J Public Health 2009;33:270-5.

35. Bell CA, Davies L, Finch M, et al. An implementation intervention to encourage healthy eating in centre-based childcare services: impact of the Good for Kids Good for Life program. Public Health Nutr 2014:1-10.

36. Wyse R, Wolfenden L, Campbell E, et al. A cluster randomised trial of a telephone based intervention for parents to increase fruit and vegetable consumption in their 3- to 5-year-old children: study protocol. BMC Public Health 2010;10:216.

37. Finch $\mathrm{M}$, Wolfenden L, Morgan $\mathrm{PJ}$, et al. A cluster randomised trial to evaluate a physical activity intervention among 3-5-year-old children attending long day care services: study protocol. BMC Public Health 2010;10:534.

38. RibisI KM, Walton MA, Mowbray CT, et al. Minimizing participant attrition in panel studies through the use of effective retention and tracking strategies: review and recommendations. Eval Program Plann 1996;19:1-25.

39. Australian Government Department of Health and Ageing. Australian guide to healthy eating. Canberra: Australian Government Department of Health and Ageing, 2013

40. Australian Government Department of Health and Ageing. National physical activity recommendations for children 0-5 years olds. Canberra: Australian Government Department of Health and Ageing, 2010.

41. Pagnini $\mathrm{D}$, Wilkenfeld $\mathrm{R}$, King $\mathrm{L}$, et al. The weight of opinion: the early childhood sectors perceptions about childhood overweight and obesity. Sydney: Centre for Overweight and Obesity, 2006.

42. Grimshaw JM, Eccles MP, Lavis JN, et al. Knowledge translation of research findings. Implement Sci 2012;7:50.

43. Grimshaw JM, Thomas RE, Maclennan G, et al. Effectiveness and efficiency of guideline dissemination and implementation strategies. Health Technol Assess 2004;8:iii--iv, 1-72.

44. Grimshaw JM, Shirran L, Thomas R, et al. Changing provider behavior: an overview of systematic reviews of interventions. Med Care 2001;39:112-45.

45. Bero LA, Grilli R, Grimshaw JM, et al. Closing the gap between research and practice: an overview of systematic reviews of interventions to promote the implementation of research findings. BMJ 1998;317:465-8.
46. Yamey G. Scaling Up global health interventions: a proposed framework for success. PLoS Med 2011;8:6.

47. Rohrbach LA, Grana R, Sussman S, et al. Type II translation: transporting prevention interventions from research to real-world settings. J Educ Eval Health Prof 2006;29:302-33.

48. Soumerai SB, Avorn J. Principles of educational outreach ('academic detailing') to improve clinical decision making. J Am Med Assoc 1990;263:549-56

49. Rohrbach LA, Graham JW, Hansen WB. Diffusion of a school-based substance abuse prevention program: Predictors of program implementation. Prev Med 1993;22:237-60.

50. Grol R, Wensing M. What drives change? Barriers to and incentives for achieving evidence-based practice. Med J Aust 2004;180: S57-60.

51. Jurgis K, Calkins E, Feather J. Enhancing Physician adoption of practice guidelines: dissemination of influenza vaccination guideline using a small- group consensus process. Arch Intern Med 1995; 155:625-32.

52. Oxman AD, Thomson MA, Davis DA, et al. No magic bullets: a systematic review of 102 trials of interventions to improve professional practice. Can Med Assoc J 1995;153:1423-31.

53. Forsetlund L, Bjørndal A, Rashidian A, et al. Continuing education meetings and workshops: effects on professional practice and health care outcomes. Cochrane Database Syst Rev 2009;(2):CD003030.

54. Read C, Kleiner B. Which training methods are effective? Manag Dev Rev 1996;9:24-9.

55. Joyce B, Showers B. Improving inservice training: the messages of research. Educ Leadersh 1980;37:379-85

56. O'Brien M, Rogers S, Jamtvedt G, et al. Educational outreach visits: effects on professional practice and health care outcomes. Cochrane Database Syst Rev 2007;(4):CD000409.

57. Alvero A, Bucklin BR, Austin A. An objective review of the effectiveness and essential characteristics of performance feedback in organizational settings (1985-1998). J Organ Behav Manage 2001;21:1-28.

58. Ivers N, Jamtvedt G, Flottorp S, et al. Audit and feedback: effects on professional practice and healthcare outcomes. Cochrane Database Syst Rev 2012;6:CD000259.

59. Noar SM, Benac CN, Harris MS. Does tailoring matter? meta-analytic review of tailored print health behavior change interventions. Psychol Bull 2007;133:673-93.

60. Damschroder LJ, Aron DC, Keith RE, et al. Fostering implementation of health services research findings into practice: a consolidated framework for advancing implementation science. Implement Sci 2009;4:50.

61. Sanders MR, Cann W, Markie-Dadds C. The Triple P-Positive Parenting Programme: a universal population-level approach to the prevention of child abuse. Child Abuse Rev 2003;12:155-71.

62. Ammerman AS, Ward DS, Benjamin SE, et al. An intervention to promote healthy weight: Nutrition and Physical Activity Self-Assessment for Child Care (NAP SACC) theory and design. Prev Chronic Dis 2007;4:A67.

63. Benjamin SE, Neelon B, Ball SC, et al. Reliability and validity of a nutrition and physical activity environment self-assessment for childcare. Int J Behav Nutr Phys Act 2007;4:1-10.

64. Henderson KE, Grode GM, Middleton AE, et al. Validity or a measure to assess the child-care nutrition and physical activity environment. J Am Diet Assoc 2011;111:1306-13.

65. Porta N, Bone C, Cobo E. Discordance between reported intention-to-treat and per protocol analyses. J Clin Epidemiol 2007;60:663-9.

66. White IR, Horton NJ, Carpenter J, et al. Strategy for intention to treat analysis in randomised trials with missing outcome data. BMJ 2011;342:910-12. 